\title{
THE SELECTIVE DECAY PRINCIPLE FOR BAROTROPIC GEOPHYSICAL FLOWS
}

\author{
ANDREW MAJDA* AND XIAOMING WANG ${ }^{\dagger}$
}

\begin{abstract}
We prove rigorously here the selective decay principle for barotropic geophysical flows. In particular we proved that the long time behavior of the solutions after renormalization is the superposition of a shear flow and a Rossby wave, and the role of hyper-viscosity is to enhance the selective decay process and the role of beta plane is to generate Rossby waves.
\end{abstract}

The purpose of this article is to study the long time behavior of the following freely decaying barotropic quasi-geostrophic equations

$$
\frac{\partial \Delta \psi}{\partial t}+\beta \frac{\partial \psi}{\partial x}+J(\psi, \Delta \psi)=\mathcal{D}(\Delta) \psi
$$

where

$$
\mathcal{D}(\Delta)=\sum_{j=1}^{k} d_{j}(-\Delta)^{j},
$$

is the dissipation operator with

$$
d_{j} \geq 0, \text { and } \sum_{j=2}^{k} d_{j}>0
$$

i.e., we assume the presence of Newtonian viscosity and/or hyper-viscosity.

It is apparent that all solutions converge to zero as time approaches infinity. However we would like to study in detail how solutions decay. We are particularly interested in the emergence of large scale structures, an interesting phenomena that frequently occurs in geophysical dynamics.

Numerical investigation of the evolution of coherent structures for freely decaying 2-D Navier-Stokes flows indicated that the enstrophy decay much more rapidly than the energy (see for instance Matthaeus et al 1991, Montgomery et al 1993, Holen 1995, Majda and Holen 1998). This suggests that one might find a suitable intermediate time scale over which the energy changes slightly so as to be regarded as nearly conserved, while the enstrophy sweeps down much more sharply. This lead physicists to hypothesize the following selection principle to characterize the large time asymptotic states of the flow

Physicist's Selective Decay Principle: After a long time, solutions of the quasigeostrophic equations and/or the two-dimensional incompressible Navier-Stokes equations approach those states which minimize the enstrophy for a given energy.

The appeal of such principle is that it reduces the calculation of the asymptotic states of the quasi-geostrophic equations and/or the Navier-Stokes system to a simpler problem in the calculus of variations. However, from the mathematical point of view the selective decay principle stated above is somewhat imprecise; the solution of the quasi-geostrophic equations and/or the Navier-Stokes equations may not approach only a minimizing state, but rather some critical point of the enstrophy. On the other

\footnotetext{
*Courant Institute of Mathematical Sciences, New York University, U.S.A.

†Department of Mathematics, Iowa State University, U.S.A.
} 
hand, the minimizing state is the only stable state. In any event, we include in the discussion all rather than just the enstrophy minimizers at constant energy.

For conciseness we refer to such a critical point of the enstrophy at constant energy as a selective decay state. We do not know a priori that selective decay states, so defined, are bona fide solutions to or invariant under the underlying equations. At the moment, a selective decay state is just a velocity or vorticity profile satisfying the variational principle. The selective decay hypothesis is that arbitrary initial velocity fields somehow approach the flow configuration of a selective decay state in the long time limit. It is hard to imagine the selective decay principle being valid unless the selective decay states turned out to be invariant under the Navier-Stokes equations and/or the quasi-geostrophic equations. More precisely, we are interested in asking if under time evolution via the quasi-geostrophic equations or the Navier-Stokes equations, a selective decay state continues to minimize the enstrophy at its energy level at later times. Such a property will at least render the Selective Decay Principle meaningful.

If such selective states are invariant under the barotropic quasi-geostrophic dynamics, we then need to check if an arbitrary solution converge to some selective state. This is the key part of the selective decay principle. We also need to explain the numerical fact that all flows converge to the largest coherent structure allowed by the geometry. This is most likely to be explained using a stability argument. We also need to identify the geophysical effects (in our case it is the beta plane and the artificial hyper-viscosity) since we are interested in geophysical applications.

To summaries, we have the following issues

- Invariance. This is the one that make the selective decay state meaningful

- Convergence. This is needed to justify the the selective decay principle.

- Stability. This is useful in interpreting the numerical results which indicates all flows converge to some ground states.

- $\beta$-plane effect, hyper-viscosity effect. This is useful since we would like to identify the geophysical effects.

For the case of Navier-Stokes equations (no beta-plane, no hyper-viscosity) the selective decay phenomena were studied by Matthaeus et al 1991, Montgomery et al 1993, Holen 1996, A. Majda and M. Holen 1998, C. Foias and J-C. Saut 1984 among others.

Next we proceed to compute the selective decay states. The computation is a simple application of the Lagrange multiplier method.

Recall that the enstrophy functional is defined as

$$
\mathcal{E}(\vec{v})=\frac{1}{2}\|\omega\|_{0}^{2}=\frac{1}{2}\|\Delta \psi\|_{0}^{2}
$$

and the energy functional is defined as

$$
E(\vec{v})=\frac{1}{2}\|\vec{v}\|_{0}^{2}=\frac{1}{2}\left\|\nabla^{\perp} \psi\right\|_{0}^{2}
$$

The variational problem we have is to minimize $\mathcal{E}$ with the following three constraints:

1. Incompressibility: $\nabla \cdot \vec{v}=0$

2. Zero mean flow: $\int_{T^{2}} \vec{v}=0$

3. Energy constraint: $E(\vec{v})=E^{\prime}$, 
where $T^{2}=[0,2 \pi] \times[0,2 \pi]$

According to the Lagrange multiplier method we deduce the following simultaneous functional relations

$$
\begin{aligned}
& E\left(\vec{v}_{\star}\right)=E^{\prime} \\
& \left.\frac{\delta \mathcal{E}}{\delta \vec{v}}\right|_{\vec{v}_{\star}}=\left.\Lambda \frac{\delta E}{\delta \vec{v}}\right|_{\vec{v}_{\star}} \\
& \nabla \cdot \vec{v}_{\star}=0 \\
& \int_{T^{2}} \vec{v}=0,
\end{aligned}
$$

The derivatives of the quadratic energy and enstrophy functionals are

$$
\begin{aligned}
& \frac{\delta E}{\delta \vec{v}}=\vec{v} \\
& \frac{\delta \mathcal{E}}{\delta \vec{v}}=-\Delta \vec{v} .
\end{aligned}
$$

Hence we end up with a simultaneous system

$$
\begin{aligned}
E\left(\vec{v}_{\star}\right) & =E^{\prime} \\
\Delta \vec{v}_{\star} & =-\Lambda \vec{v}_{\star} \\
\nabla \cdot \vec{v}_{\star} & =0 \\
\int_{T^{2}} \vec{v}_{\star} & =0 .
\end{aligned}
$$

This implies that stream function $\psi_{\star}$ for the selective decay state must satisfy

$$
\begin{aligned}
E\left(\nabla^{\perp} \psi_{\star}\right) & =E^{\prime} \\
-\Delta \psi_{\star} & =\Lambda \psi_{\star} .
\end{aligned}
$$

Hence it must be one of the eigenfunctions of the Laplace operator.

It is interesting to notice that such eigenvalue-eigenfunction problems also emerge in the classical energy-enstrophy statistical mechanics in predicting the most probable states (see for instance R. Kraichnan and D. Montgomery 1980, A. Majda and M. Holen 1998 among others).

The eigenfunctions of the Laplacian in the periodic setting can be easily calculated as the generalized Taylor vortices:

$$
\begin{aligned}
\psi_{j} & =\sum_{|\vec{k}|^{2}=\Lambda_{j}} A_{\vec{k}} e^{i \vec{k} \cdot \vec{x}}+c . c \\
\vec{v}_{j} & =\nabla^{\perp} \psi_{j}
\end{aligned}
$$

where $\Lambda_{j}=|\vec{k}|^{2}, \vec{k} \in Z^{2}$ are the eigenvalues

Recall that

$$
\mathcal{E}\left(\vec{v}_{j}\right)=\Lambda_{j} E\left(\vec{v}_{j}\right)
$$

hence we may conclude

1. The ground state is the actual minimizer of the enstrophy with given energy. Other Taylor vortices are saddle points. 
2. All flows will approach a Taylor vortex of the lowest eigenvalue $\Lambda=\Lambda_{\star}$ permitted by the symmetries if the physicists' selective decay principle is true.

We check the first issue for selective decay states, i.e., the invariance of these states under the barotropic quasi-geostrophic dynamics.

The first thing we notice is that the nonlinear term drops for selective decay states, i.e.,

$$
J(\psi, \Delta \psi)=0
$$

since

$$
\Delta \psi=-\Lambda_{j} \psi
$$

Hence we end up with a linear equation

$$
\frac{\partial \omega}{\partial t}+\beta \frac{\partial \psi}{\partial x}=\mathcal{D}\left(-\Lambda_{j}\right) \psi
$$

The solutions can be computed easily as

$$
\psi(t)=\sum_{|\vec{k}|^{2}=\Lambda_{j}} A_{\vec{k}}(0) e^{i \vec{k} \cdot \vec{x}-i k_{1} t \frac{\beta}{\Lambda_{j}}} e^{-\frac{\mathcal{D}\left(-\Lambda_{j}\right)}{\Lambda_{j}} t}
$$

It is then reasonable to speculate, based on this representation, that

- The $\beta$ plane effect is to generate Rossby wave

- The Rossby waves degenerate into generalized Taylor vortices at vanishing $\beta$ effect

Of course these statements still need to be verified.

Since we are studying freely decaying flows, it is natural to study the Normalized velocity field $\tilde{\vec{v}}(t)$

$$
\tilde{\vec{v}}(t)=\frac{\vec{v}(t)}{\|\vec{v}(t)\|_{0}}
$$

or the normalize (in $H^{1}$ ) stream function

$$
\tilde{\psi}(t)=\frac{\psi(t)}{\|\vec{v}(t)\|_{0}}
$$

Since the numerical results suggest the consistently more rapid decay of the enstrophy over the energy, we introduce the Dirichlet quotient $\Lambda(t)$ :

$$
\Lambda(t)=\frac{\mathcal{E}(t)}{E(t)}
$$

We now state our main result

TheOREM 1 (Math Form). For arbitrary initial data, the Dirichlet quotient $\Lambda(t)$ monotonically decreases to an eigenvalue $\Lambda_{j}$ of the Laplace operator, i.e.

$$
\lim _{t \rightarrow \infty} \Lambda(t)=\Lambda_{j}
$$


There exists a solution $\vec{v}_{j}(t)$ of the linearized barotropic quasi-geostrophic equation with motion restricted on the $j^{\text {th }}$ shell (this implies that it is a superposition of a shear flow and a Rossby wave) such that

$$
\left\|\tilde{\vec{v}}(t)-\vec{v}_{j}(t)\right\|_{0} \rightarrow 0 \text { as } t \rightarrow \infty
$$

The Rossby wave degenerates into a generalized Taylor vortices in the absence of the geophysical $\beta$ plane effect.

If the numerical results are true, we should have the decay of the Dirichlet quotient since enstrophy decay faster than energy

$$
\frac{d \Lambda(t)}{d t} \leq 0
$$

This is indeed the case. In fact we have

LEMMA 1.

$$
\begin{aligned}
\frac{d \Lambda(t)}{d t}= & -\frac{1}{E(t)} \sum_{j=2}^{k} \sum_{l=0}^{j-2} d_{j} \Lambda^{l}(t) \\
& \left\|(-\Delta)^{\frac{j+1-l}{2}} \psi-\Lambda(t)(-\Delta)^{\frac{j-1-l}{2}} \psi\right\|_{0}^{2} .
\end{aligned}
$$

In particular, $\frac{d \Lambda(t)}{d t} \leq 0$ with equality obtained only for the selective decay states.

It seems worthwhile to point out two simple consequences of this lemma

- Hyper-viscosity enhances selective decay process,

- Ekman damping $d_{1}$ has no selective decay effect. ${ }^{1}$

The original proof of the decay of Dirichlet quotient in the presence of hyperviscosity is due to Z.P. Xin (1998). We present here an alternative proof.

In order to prove the lemma, we recall the well-known optimal (independent of initial data) decay estimates for the energy and enstrophy

$$
\begin{aligned}
& E(t) \leq E(0) e^{-2 \frac{\mathcal{D}\left(-\Lambda_{1}\right)}{\Lambda_{1}} t}, \\
& \mathcal{E}(t) \leq \mathcal{E}(0) e^{-2 \frac{\mathcal{D}\left(\Lambda_{1}\right)}{\Lambda_{1}} t} .
\end{aligned}
$$

We recall the definition of fractional power of the Laplace operator. Let

$$
\psi=\sum_{\vec{k}} A_{\vec{k}} e^{i \vec{k} \cdot \vec{x}}
$$

we then define

$$
(-\Delta)^{s} \psi=\sum_{l} \sum_{|\vec{k}|^{2}=\Lambda_{l}} \Lambda_{l}^{s} A_{\vec{k}} e^{i \vec{k} \cdot \vec{x}}
$$

It is easy to derive the dynamic equations for energy and enstrophy:

$$
\begin{aligned}
& \frac{d E(t)}{d t}=-\sum_{j=1}^{k} d_{j}\left\|(-\Delta)^{\frac{j}{2}} \psi\right\|_{0}^{2}, \\
& \frac{d \mathcal{E}(t)}{d t}=-\sum_{j=1}^{k} d_{j}\left\|(-\Delta)^{\frac{j+1}{2}} \psi\right\|_{0}^{2} .
\end{aligned}
$$

\footnotetext{
${ }^{1}$ If we take into consideration the effect of stratification in our barotropic model, the Ekman damping turns out has selective decay effect.
} 
We then deduce the equation satisfied by the Dirichlet quotient:

$$
\begin{aligned}
\frac{d \Lambda(t)}{d t} & =\frac{d}{d t}\left(\frac{\mathcal{E}(t)}{E(t)}\right) \\
& =\frac{\dot{\mathcal{E}}(t)}{E(t)}-\frac{\mathcal{E}(t) \dot{E}(t)}{E^{2}(t)} \\
& =\frac{1}{E(t)}(\dot{\mathcal{E}}(t)-\Lambda(t) \dot{E}(t)) \\
& =-\frac{1}{E(t)}\left(\sum_{j=1}^{k} d_{j}\left\|(-\Delta)^{\frac{j+1}{2}} \psi\right\|_{0}^{2}-\Lambda(t) \sum_{j=1}^{k} d_{j}\left\|(-\Delta)^{\frac{j}{2}} \psi\right\|_{0}^{2}\right) \\
& =-\frac{1}{E(t)} \sum_{j=1}^{k} d_{j}\left(\left\|(-\Delta)^{\frac{j+1}{2}} \psi\right\|_{0}^{2}-\Lambda(t)\left\|(-\Delta)^{\frac{j}{2}} \psi\right\|_{0}^{2}\right) .
\end{aligned}
$$

We observe (this is the key part of the proof of this lemma) that

$$
\begin{aligned}
& \left\|(-\Delta)^{\frac{j+1}{2}} \psi\right\|_{0}^{2}-\Lambda(t)\left\|(-\Delta)^{\frac{j}{2}} \psi\right\|_{0}^{2} \\
= & \left\|(-\Delta)^{\frac{j+1}{2}} \psi-\Lambda(t)(-\Delta)^{\frac{j-1}{2}} \psi\right\|_{0}^{2} \\
& +2 \Lambda(t)\left((-\Delta)^{\frac{j+1}{2}} \psi,(-\Delta)^{\frac{j-1}{2}} \psi\right) \\
& -\Lambda^{2}(t)\left\|(-\Delta)^{\frac{j-1}{2}} \psi\right\|_{0}^{2}-\Lambda(t)\left\|(-\Delta)^{\frac{j}{2}} \psi\right\|_{0}^{2} \\
= & \left\|(-\Delta)^{\frac{j+1}{2}} \psi-\Lambda(t)(-\Delta)^{\frac{j-1}{2}} \psi\right\|_{0}^{2} \\
& +2 \Lambda(t)\left((-\Delta)^{\frac{j}{2}} \psi,(-\Delta)^{\frac{j}{2}} \psi\right) \\
& -\Lambda^{2}(t)\left\|(-\Delta)^{\frac{j-1}{2}} \psi\right\|_{0}^{2}-\Lambda(t)\left\|(-\Delta)^{\frac{j}{2}} \psi\right\|_{0}^{2} \\
= & \left\|(-\Delta)^{\frac{j+1}{2}} \psi-\Lambda(t)(-\Delta)^{\frac{j-1}{2}} \psi\right\|_{0}^{2} \\
& +\Lambda(t)\left(\left\|(-\Delta)^{\frac{j}{2}} \psi\right\|_{0}^{2}-\Lambda(t)\left\|(-\Delta)^{\frac{j-1}{2}} \psi\right\|_{0}^{2}\right),
\end{aligned}
$$

and

$$
\|-\Delta \psi\|_{0}^{2}-\Lambda(t)\left\|(-\Delta)^{\frac{1}{2}} \psi\right\|_{0}^{2}=0
$$

thus

$$
\begin{aligned}
& \left\|(-\Delta)^{\frac{j+1}{2}} \psi\right\|_{0}^{2}-\Lambda(t)\left\|(-\Delta)^{\frac{j}{2}} \psi\right\|_{0}^{2} \\
= & \sum_{l=0}^{j-2} \Lambda^{l}(t)\left\|(-\Delta)^{\frac{j+1-l}{2}} \psi-\Lambda(t)(-\Delta)^{\frac{j-1-l}{2}} \psi\right\|_{0}^{2},
\end{aligned}
$$

This completes the proof of the lemma.

With this lemma in hand, we deduce the following

1. $\frac{d \Lambda(t)}{d t} \leq 0, \frac{d \Lambda(t)}{d t}=0$ if and only if $\vec{v}=\nabla^{\perp} \psi_{j}$ is one of the selective decay states, and $\Lambda(t)=\Lambda_{j}$ for all succeeding times.

2. $\Lambda(t) \geq \Lambda_{1}=1$ by Poincaré inequality

3. Hence

$$
\lim _{t \rightarrow \infty} \Lambda(t)=\Lambda_{*} \geq \Lambda_{1}
$$

must exist. 
The natural question to ask is then if $\Lambda_{*}=\Lambda_{j}$ for some $j$, or if $\Lambda_{*}$ is one of the eigenvalues of the laplacian.

Without loss of generality we assume $d_{2}>0$. Notice

$$
\begin{aligned}
& 2 d_{2} \int_{0}^{\infty} \frac{\|(-\Delta-\Lambda(t)) \vec{v}\|_{0}^{2}}{\|\vec{v}\|_{0}^{2}} d t \\
= & 2 d_{2} \int_{0}^{\infty} \frac{\left\|(-\Delta)^{\frac{3}{2}} \psi-\Lambda(t)(-\Delta)^{\frac{1}{2}} \psi\right\|_{0}^{2}}{\left\|\nabla^{\perp} \psi\right\|_{0}^{2}} d t \\
\leq & -\int_{0}^{\infty} \frac{d \Lambda}{d t} \\
= & \Lambda(0)-\lim _{t \rightarrow \infty} \Lambda(t) \\
= & \Lambda(0)-\Lambda_{\star} \\
< & \infty
\end{aligned}
$$

Let

$$
\delta \lambda=\min _{j}\left(\left|\Lambda_{\star}-\Lambda_{j}\right|\right)
$$

we have, for large enough $t$

$$
\begin{aligned}
\frac{\|(-\Delta-\Lambda(t)) \vec{v}\|_{0}^{2}}{\|\vec{v}\|_{0}^{2}} & =\|(-\Delta-\Lambda(t)) \tilde{\vec{v}}\|_{0}^{2} \\
& =\sum_{j} \sum_{|\vec{k}|^{2}=\Lambda_{j}}\left(\Lambda_{j}-\Lambda(t)\right)^{2} \Lambda_{j}\left|A_{\vec{k}}\right|^{2} 4 \pi^{2} \\
& \geq \pi^{2}\left(\min _{j}\left(\left|\Lambda_{\star}-\Lambda_{j}\right|\right)\right)^{2} \\
& =\pi^{2}(\delta \lambda)^{2}
\end{aligned}
$$

This contradicts the integrability of $\frac{\|(-\Delta-\Lambda(t)) \vec{v}\|_{0}^{2}}{\|\vec{v}\|_{0}^{2}}$ if $\delta \lambda>0$.

We are now able to prove a weak version of the selective decay theorem. Namely we will prove that there is a time sequence $\left\{t_{j}\right\} \rightarrow \infty$ such that $\vec{v}\left(t_{j}\right)$ converge to one of the selective decay states. We first notice that we have the following bound on the $H^{1}$ norm of the normalized flow:

$$
\|\tilde{\vec{v}}(t)\|_{0}^{2}+\|\nabla \tilde{\vec{v}}(t)\|_{0}^{2}=1+\Lambda(t) \leq 1+\Lambda(0)
$$

This together with the integrability of the function $\|(-\Delta-\Lambda(t)) \tilde{\vec{v}}(t)\|_{0}^{2}$ implies that we can take a sequence $\left\{t_{j}\right\}$ such that

$$
\begin{gathered}
\tilde{\vec{v}}\left(t_{j}\right) \rightarrow \vec{v}_{\star} \text { weakly } \in H^{1}, \\
\left\|\tilde{\vec{v}}\left(t_{j}\right)\right\|_{0}=1=\left\|\vec{v}_{\star}\right\|_{0}
\end{gathered}
$$

and

$$
\left(-\Delta-\Lambda\left(t_{j}\right)\right) \tilde{\vec{v}}\left(t_{j}\right) \rightarrow 0
$$


Next we show that $\vec{v}_{\star}$ is a selective decay state.

For this purpose let $\phi$ be a test function (in the doubly periodic case it is any trigonometric polynomial).

We check the divergence free property first

$$
\left(\nabla \cdot \vec{v}_{\star}, \phi\right)_{0} \equiv-\left(\vec{v}_{\star}, \nabla \phi\right)_{0}=-\lim _{t_{j} \rightarrow \infty}\left(\tilde{\vec{v}}\left(t_{j}\right), \nabla \phi\right)_{0}=\lim _{t_{j}}\left(\nabla \cdot \tilde{\vec{v}}\left(t_{j}\right), \phi\right)_{0}=0 .
$$

The eigen equation can be checked in a similar fashion

$$
\begin{aligned}
\left(\left(-\Delta-\Lambda_{\star}\right) \vec{v}_{\star}, \phi\right)_{0} & \equiv\left(\vec{v}_{\star},\left(-\Delta-\Lambda_{\star}\right) \phi\right)_{0} \\
& =\lim _{t_{j}}\left(\tilde{\vec{v}}\left(t_{j}\right),\left(-\Delta-\Lambda\left(t_{j}\right)\right) \phi\right)_{0} \\
& =\lim _{t_{j}}\left(\left(-\Delta-\Lambda\left(t_{j}\right)\right) \tilde{\vec{v}}\left(t_{j}\right), \phi\right)_{0} \\
& =0
\end{aligned}
$$

thanks to the integrability of $(-\Delta-\Lambda(t)) \tilde{\vec{v}}(t)$ and our choice of the subsequence $\left\{t_{j}\right\}$. The mean zero property can be verified easily as

$$
\int_{T^{2}} \vec{v}_{\star}=\lim _{j \rightarrow \infty} \int_{T^{2}} \tilde{\vec{v}}\left(t_{j}\right)=0
$$

This proves a weak version of the selective decay principle which partially verifies the physicist's version of selective decay. Notice this weak version does not indicate the role of $\beta$ plane, nor the connections betwen the limiting behaviors of different sequences. These details are furnished by our main theorem.

In order to prove our theorem we assume Newtonian viscosity for simplicity i.e.

$$
d_{2}=\nu>0, \text { and } d_{j}=0 \forall j \neq 2
$$

Our intuition is that the motion is concentrated on the $j_{t h}$ shell.

To verify our intuition it is useful to introduce the following projection operators

- $P_{\Lambda_{j}}$ : projection onto the $j^{\text {th }}$ shell;

- $Q_{\Lambda_{j}^{-}}$: projection onto the lower modes;

- $Q_{\Lambda_{j}^{+}}$: projection onto the higher modes.

The normalized flow is

$$
\tilde{\psi}=\frac{\psi}{\|v\|_{0}}
$$

Our main theorem is now equivalent to the combination of the following three properties

- Decay of the lower modes

$$
Q_{\Lambda_{j}^{-}} \tilde{\psi}(t) \rightarrow 0 \quad \text { in } \quad H^{1}
$$

- Decay of the higher modes

$$
Q_{\Lambda_{j}^{+}} \tilde{\psi}(t) \rightarrow 0 \text { in } H^{1}
$$

- Linear dynamics on the $j^{\text {th }}$ shell, i.e., there exists $\tilde{\xi}_{j}$ (solution to the linear barotropic quasi-geostrophic equation) such that

$$
P_{\Lambda_{j}} \tilde{\psi}(t)-\tilde{\xi}_{j}(t) \rightarrow 0 \quad \text { in } \quad H^{1}
$$


To start with, we recall the well-known optimal (initial data dependent!) decay estimates for energy and enstrophy.

Indeed, thanks to the energy equation

$$
\frac{1}{2} \frac{d}{d t}\|\vec{v}(t)\|_{0}^{2}+\nu\|\omega(t)\|_{0}^{2}=\frac{1}{2} \frac{d}{d t}\|\vec{v}(t)\|_{0}^{2}+\nu \Lambda(t)\|\vec{v}(t)\|_{0}^{2}=0 .
$$

we have

$$
\frac{1}{2} \frac{d}{d t}\|\vec{v}(t)\|_{0}^{2}+\nu \Lambda_{j}\|\vec{v}(t)\|_{0}^{2} \leq 0
$$

since $\Lambda(t)$ is non-increasing and the limit is $\Lambda_{j}$. This implies

$$
\|\vec{v}(t)\|_{0} \leq\left\|\vec{v}\left(t_{0}\right)\right\|_{0} e^{-\nu \Lambda_{j}\left(t-t_{0}\right)} .
$$

Thus the enstrophy is bounded by

$$
\|\omega(t)\|_{0} \leq\left\|\omega\left(t_{0}\right)\right\|_{0} e^{-\nu \Lambda_{j}\left(t-t_{0}\right)}
$$

since

$$
\begin{aligned}
\|\omega(t)\|_{0}^{2} & =\Lambda(t)\|\vec{v}(t)\|_{0}^{2} \\
& \leq \Lambda\left(t_{0}\right)\left\|\vec{v}\left(t_{0}\right)\right\|_{0}^{2} e^{-2 \nu \Lambda_{j}\left(t-t_{0}\right)} \\
& =\left\|\omega\left(t_{0}\right)\right\|_{0}^{2} e^{-2 \nu \Lambda_{j}\left(t-t_{0}\right)} .
\end{aligned}
$$

A lower bound on the energy decay rate can be derived as well. In deed, since the Dirichlet quotient is non-increasing we have, thanks to the energy equation,

$$
\frac{1}{2} \frac{d}{d t}\|\vec{v}(t)\|_{0}^{2}+\nu \Lambda\left(t_{0}\right)\|\vec{v}(t)\|_{0}^{2} \geq 0, \forall t \geq t_{0}
$$

Hence we have

$$
\|\vec{v}(t)\|_{0}^{2} \geq\left\|\vec{v}\left(t_{0}\right)\right\|_{0}^{2} e^{-2 \nu \Lambda\left(t_{0}\right)\left(t-t_{0}\right)}, \forall t \geq t_{0}
$$

We may also derive a decay rate for $\nabla \Delta \psi$.

For this purpose we multiply the barotropic quasi-geostrophic equation by $-\Delta^{2} \psi$ and integrate over $T^{2}$. Notice for the nonlinear term we have

$$
\begin{aligned}
\left|\int J(\psi, \Delta \psi) \Delta^{2} \psi\right| & \leq\|\nabla \psi\|_{L^{\infty}}\left\|\nabla^{\perp} \Delta \psi\right\|_{0}\left\|\Delta^{2} \psi\right\|_{0} \\
& \leq c\|\nabla \psi\|_{0}^{\frac{1}{2}}\left\|\nabla^{\perp} \Delta \psi\right\|_{0}^{\frac{3}{2}}\left\|\Delta^{2} \psi\right\|_{0}^{2} \\
& \leq c\|\nabla \psi\|_{0}^{\frac{1}{2}}\|\Delta \psi\|_{0}^{\frac{3}{4}}\left\|\Delta^{2} \psi\right\|_{0}^{\frac{7}{4}} \\
& \leq \frac{\nu}{2}\left\|\Delta^{2} \psi\right\|_{0}^{2}+c\|\nabla \psi(t)\|_{0}^{4}\|\Delta \psi(t)\|_{0}^{6} \\
& \leq \frac{\nu}{2}\left\|\Delta^{2} \psi\right\|_{0}^{2}+c\left\|\nabla \psi\left(t_{0}\right)\right\|_{0}^{4}\left\|\Delta \psi\left(t_{0}\right)\right\|_{0}^{6} e^{-10 \nu \Lambda_{j}\left(t-t_{0}\right)}
\end{aligned}
$$

we we employed classical Sobolev imbedding, Agmon inequality, interpolation inequality, Hölder inequality and our decay estimates on the energy and enstrophy. Thus we deduce

$$
\frac{d}{d t}\|\nabla \Delta \psi\|_{0}^{2}+\nu\left\|\Delta^{2} \psi\right\|_{0}^{2} \leq c\left\|\nabla \psi\left(t_{0}\right)\right\|_{0}^{4}\left\|\Delta \psi\left(t_{0}\right)\right\|_{0}^{6} e^{-10 \nu \Lambda_{j}\left(t-t_{0}\right)}
$$


Notice, by interpolation inequality,

$$
\begin{aligned}
\left\|\Delta^{2} \psi\right\|_{0}^{2} & \geq\|\nabla \Delta \psi\|_{0}^{2} \frac{\|\nabla \Delta \psi\|_{0}^{2}}{\|\Delta \psi\|_{0}^{2}} \\
& \geq\|\nabla \Delta \psi\|_{0}^{2} \frac{\|\Delta \psi\|_{0}^{2}}{\|\nabla \psi\|_{0}^{2}} \\
& =\Lambda(t)\|\nabla \Delta \psi\|_{0}^{2} \\
& \geq \Lambda_{j}\|\nabla \Delta \psi\|_{0}^{2} .
\end{aligned}
$$

Hence

$$
\frac{d}{d t}\|\nabla \Delta \psi\|_{0}^{2}+\nu \Lambda_{j}\|\nabla \Delta \psi\|_{0}^{2} \leq c\left\|\nabla \psi\left(t_{0}\right)\right\|_{0}^{4}\left\|\Delta \psi\left(t_{0}\right)\right\|_{0}^{6} e^{-10 \nu \Lambda_{j}\left(t-t_{0}\right)}
$$

Applying a Gronwall type inequality we deduce

$$
\|\nabla \Delta \psi(t)\|_{0}^{2} \leq e^{-\nu \Lambda_{j}\left(t-t_{0}\right)}\left\|\nabla \Delta \psi\left(t_{0}\right)\right\|_{0}^{2}+c\left\|\nabla \psi\left(t_{0}\right)\right\|_{0}^{4}\left\|\Delta \psi\left(t_{0}\right)\right\|_{0}^{6} e^{-\nu \Lambda_{j}\left(t-t_{0}\right)}
$$

This is the decay rate that we will use in the sequel.

In order to carry out our three tasks regarding the normalized stream function it is useful to recall the equation satisfied by the normalized (in $H^{1}$ ) stream function $\tilde{\psi}$. It is easy to check that

$$
\frac{d}{d t} \Delta \tilde{\psi}(t)+\beta \frac{\partial \tilde{\psi}}{\partial x}+\|\vec{v}(t)\|_{0} J(\tilde{\psi}, \Delta \tilde{\psi})-\nu \Delta^{2} \tilde{\psi}-\nu \Lambda(t) \Delta \tilde{\psi}(t)=0 .
$$

We notice that the nonlinear effect decays as the time evolves for this normalized problem.

We start the process with the simplest task among the three, namely the decay of higher modes. This is intuitively clear since we anticipate that higher modes decay faster if the nonlinear effect is negligible.

Notice for the nonlinear term we have

$$
\begin{aligned}
\left|\int J(\tilde{\psi}, \Delta \tilde{\psi}) Q_{\Lambda_{j}^{+}} \tilde{\psi}\right| & =\left|\int J\left(\tilde{\psi}, Q_{\Lambda_{j}^{+}} \tilde{\psi}\right) \Delta \tilde{\psi}\right| \\
& \leq\|\nabla \tilde{\psi}\|_{L^{4}}\left\|\nabla^{\perp} Q_{\Lambda_{j}^{+}} \tilde{\psi}\right\|_{L^{4}}\|\Delta \tilde{\psi}\|_{0} \\
& \leq c\|\nabla \tilde{\psi}\|_{0}^{\frac{1}{2}}\|\nabla \tilde{\psi}\|_{H^{1}}^{\frac{1}{2}}\left\|\nabla^{\perp} Q_{\Lambda_{j}^{+}} \tilde{\psi}\right\|_{0}^{\frac{1}{2}}\left\|\nabla^{\perp} Q_{\Lambda_{j}^{+}} \tilde{\psi}\right\|_{H^{1}}^{\frac{1}{2}}\|\Delta \tilde{\psi}\|_{0} \\
& \leq c\|\nabla \tilde{\psi}\|_{0}\|\nabla \tilde{\psi}\|_{H^{1}}\|\Delta \tilde{\psi}\|_{0} \\
& \leq c \Lambda(t) .
\end{aligned}
$$

Hence we have (since $\Lambda(t)$ is uniformly bounded),

$$
\begin{aligned}
\frac{1}{2} \frac{d}{d t}\left\|\nabla Q_{\Lambda_{j}^{+}} \tilde{\psi}\right\|_{0}^{2}+\nu\left\|\Delta Q_{\Lambda_{j}^{+}} \tilde{\psi}\right\|_{0}^{2}-\nu \Lambda(t)\left\|\nabla Q_{\Lambda_{j}^{+}} \tilde{\psi}\right\|_{0}^{2} & \leq c \Lambda(t)\|\vec{v}(t)\|_{0} \\
& \leq c e^{-\nu \Lambda_{j}\left(t-t_{0}\right)}
\end{aligned}
$$

Thanks to Poincaré inequality, we have

$$
\left\|\Delta Q_{\Lambda_{j}^{+}} \tilde{\psi}\right\|_{0}^{2} \geq \Lambda_{j+1}\left\|\nabla Q_{\Lambda_{j}^{+}} \tilde{\psi}\right\|_{0}^{2}
$$


thus

$$
\frac{1}{2} \frac{d}{d t}\left\|\nabla Q_{\Lambda_{j}^{+}} \tilde{\psi}\right\|_{0}^{2}+\nu\left(\Lambda_{j+1}-\Lambda(t)\right)\left\|\nabla Q_{\Lambda_{j}^{+}} \tilde{\psi}\right\|_{0}^{2} \leq c e^{-\nu \Lambda_{j}\left(t-t_{0}\right)}
$$

Now we take $t_{0}$ large enough so that

$$
\Lambda(t) \leq \frac{1}{2}\left(\Lambda_{j+1}+\Lambda_{j}\right), \quad \forall \quad t \geq t_{0}
$$

we have

$$
\frac{d}{d t}\left\|\nabla Q_{\Lambda_{j}^{+}} \tilde{\psi}\right\|_{0}^{2}+\nu\left(\Lambda_{j+1}-\Lambda_{j}\right)\left\|\nabla Q_{\Lambda_{j}^{+}} \tilde{\psi}\right\|_{0}^{2} \leq c e^{-\nu \Lambda_{j}\left(t-t_{0}\right)}, \quad \forall \quad t \geq t_{0}
$$

This further implies, Gronwall type inequality,

$\left\|\nabla Q_{\Lambda_{j}^{+}} \tilde{\psi}(t)\right\|_{0}^{2} \leq e^{-\nu\left(\Lambda_{j+1}-\Lambda_{j}\right)\left(t-t_{0}\right)}\left\|\nabla Q_{\Lambda_{j}^{+}} \tilde{\psi}(t)\right\|_{0}^{2}+c\left(e^{-\nu \Lambda_{j}\left(t-t_{0}\right)}+e^{-\nu\left(\Lambda_{j+1}-\Lambda_{j}\right)\left(t-t_{0}\right)}\right)$

This proves the decay of the higher modes.

We now proceed with the proof of the decay of the lower modes. This is less obvious. We employ here a Lyapunov-Perron type technique which is frequently used in the study of long time behavior of dynamical systems.

Notice for the nonlinear term we have

$$
\left|\int J(\tilde{\psi}, \Delta \tilde{\psi}) Q_{\Lambda_{j}^{-}} \tilde{\psi}\right| \leq c \Lambda(t)
$$

just as in the case of higher modes.

Hence we deduce

$$
\begin{aligned}
\frac{1}{2} \frac{d}{d t}\left\|\nabla Q_{\Lambda_{j}^{-}} \tilde{\psi}\right\|_{0}^{2}+\nu\left\|\Delta Q_{\Lambda_{j}^{-}} \tilde{\psi}\right\|_{0}^{2}-\nu \Lambda(t)\left\|\nabla Q_{\Lambda_{j}^{-}} \tilde{\psi}\right\|_{0}^{2} & \geq-c \Lambda(t)\|\vec{v}(t)\|_{0} \\
& \geq-c e^{-\nu \Lambda_{j}\left(t-t_{0}\right)}
\end{aligned}
$$

Thanks to a reversed Poincaré inequality on the range of $Q_{\Lambda_{j}^{-}}$we have

$$
\left\|\Delta Q_{\Lambda_{j}^{-}} \tilde{\psi}\right\|_{0}^{2} \leq \Lambda_{j-1}\left\|\nabla Q_{\Lambda_{j}^{-}} \tilde{\psi}\right\|_{0}^{2}
$$

We then deduce

$$
\begin{aligned}
\frac{1}{2} \frac{d}{d t}\left\|\nabla Q_{\Lambda_{j}^{-}} \tilde{\psi}\right\|_{0}^{2} & \geq \nu\left(\Lambda(t)-\Lambda_{j-1}\right)\left\|\nabla Q_{\Lambda_{j}^{-}} \tilde{\psi}\right\|_{0}^{2}-c e^{-\nu \Lambda_{j}\left(t-t_{0}\right)} \\
& \geq \nu\left(\Lambda_{j}-\Lambda_{j-1}\right)\left\|\nabla Q_{\Lambda_{j}^{-}} \tilde{\psi}\right\|_{0}^{2}-c e^{-\nu \Lambda_{j}\left(t-t_{0}\right)}
\end{aligned}
$$

or

$$
\frac{d}{d t}\left(e^{-2 \nu\left(\Lambda_{j}-\Lambda_{j-1}\right) t}\left\|\nabla Q_{\Lambda_{j}^{-}} \tilde{\psi}(t)\right\|_{0}^{2}\right) \geq-c e^{-\nu\left(3 \Lambda_{j}-2 \Lambda_{j-1}\right) t}
$$

Integrating this inequality from $t$ to $T\left(T>t>t_{0}\right)$ we obtain

$$
e^{-2 \nu\left(\Lambda_{j}-\Lambda_{j-1}\right) T}\left\|\nabla Q_{\Lambda_{j}^{-}} \tilde{\psi}(T)\right\|_{0}^{2} \geq e^{-2 \nu\left(\Lambda_{j}-\Lambda_{j-1}\right) t}\left\|\nabla Q_{\Lambda_{j}^{-}} \tilde{\psi}(t)\right\|_{0}^{2}-c e^{-\nu\left(3 \Lambda_{j}-2 \Lambda_{j-1}\right) t}
$$

or equivalently

$$
e^{-2 \nu\left(\Lambda_{j}-\Lambda_{j-1}\right)(T-t)}\left\|\nabla Q_{\Lambda_{j}^{-}} \tilde{\psi}(T)\right\|_{0}^{2} \geq\left\|\nabla Q_{\Lambda_{j}^{-}} \tilde{\psi}(t)\right\|_{0}^{2}-c e^{-\nu \Lambda_{j} t}
$$


Letting $T$ approach infinity $\left(\|\nabla \tilde{\psi}\|_{0}=1\right)$ we get

$$
\left\|\nabla Q_{\Lambda_{j}^{-}} \tilde{\psi}(t)\right\|_{0}^{2} \leq c e^{-\nu \Lambda_{j} t}
$$

This proves the decay of lower modes.

We now concentrate on the dynamics on the $j_{t h}$ shell.

For convenience we introduce the notation

$$
\psi_{j}=P_{\Lambda_{j}} \psi
$$

We notice that $\psi_{j}$ satisfies the equation

$$
-\Lambda_{j} \frac{d}{d t} \psi_{j}+\beta \frac{\partial \psi_{j}}{\partial x}+P_{\Lambda_{j}} J(\psi, \Delta \psi)=\nu \Lambda_{j}^{2} \psi_{j}
$$

or equivalently

$$
\frac{d}{d t} \psi_{j}-\frac{\beta}{\Lambda_{j}} \frac{\partial \psi_{j}}{\partial x}+\nu \Lambda_{j} \psi_{j}=e^{-\nu \Lambda_{j} t} f
$$

where

$$
f=\frac{e^{\nu \Lambda_{j} t}}{\Lambda_{j}} P_{\Lambda_{j}} J(\psi, \Delta \psi) .
$$

The equation can be written in the following form

$$
\frac{d}{d t}\left(e^{\nu \Lambda_{j} t} \psi_{j}\right)-\frac{\beta}{\Lambda_{j}} \frac{\partial\left(e^{\nu \Lambda_{j} t} \psi_{j}\right)}{\partial x}=f .
$$

It is easy to see that $f$ satisfies the following estimate

$$
\begin{aligned}
\|f(t)\|_{0} & \leq c e^{\nu \Lambda_{j} t}\|\nabla \psi\|_{L^{\infty}}\|\nabla \Delta \psi\|_{0} \\
& \leq c e^{\nu \Lambda_{j} t}\|\Delta \psi\|_{0}\left(1+\log \frac{\|\nabla \Delta \psi\|_{0}^{2}}{\Lambda_{1}\|\Delta \psi\|_{0}^{2}}\right)^{\frac{1}{2}}\|\nabla \Delta \psi\|_{0} \\
& \leq c e^{\nu \Lambda_{j} t}\|\Delta \psi\|_{0}\left(1+\log \frac{e^{-\nu \Lambda_{j} t}}{e^{-\nu \Lambda(0) t}}\right)^{\frac{1}{2}} e^{-\nu \Lambda_{j} t / 2} \\
& \leq c\left(\Lambda(0)-\Lambda_{j}\right)^{\frac{1}{2}} t^{\frac{1}{2}} e^{-\nu \Lambda_{j} t / 2}
\end{aligned}
$$

where we applied the Brezis-Gallouet inequality, the optimal decay rate for the enstrophy and the lower bound on the decay rate for energy. This estimate implies that

$$
f \in L^{1}
$$

To better understand the situation, we invoke the Fourier series representation. Let

$$
\begin{gathered}
\psi_{j}=\sum_{|\vec{k}|^{2}=\Lambda_{j}} \hat{\psi}_{\vec{k}} e^{i \vec{k} \cdot \vec{x}} \\
f=\sum_{|\vec{k}|^{2}=\Lambda_{j}} \hat{f}_{\vec{k}} e^{i \vec{k} \cdot \vec{x}}
\end{gathered}
$$


We then have

$$
\frac{d \hat{\psi}_{\vec{k}}}{d t}-\frac{i \beta k_{1}}{\Lambda_{j}} \hat{\psi}_{\vec{k}}+\nu \Lambda_{j} \hat{\psi}_{\vec{k}}=e^{-\nu \Lambda_{j} t} \hat{f}_{\vec{k}}
$$

which is equivalent to

$$
\frac{d}{d t}\left(e^{\left(\nu \Lambda_{j}-\frac{i \beta k_{1}}{\Lambda_{j}}\right) t} \hat{\psi}_{\vec{k}}\right)=e^{-\frac{i \beta k_{1}}{\Lambda_{j}} t} \hat{f}_{\vec{k}}
$$

This suggest that we make a mode dependent phase shift and introduce the following new variable

$$
\phi_{j}=\sum_{|\vec{k}|^{2}=\Lambda_{j}} \hat{\psi}_{\vec{k}} e^{\left(\nu \Lambda_{j}-\frac{i \beta k_{1}}{\Lambda_{j}}\right) t} e^{i \vec{k} \cdot \vec{x}}
$$

The new variable satisfies the equation

$$
\frac{d}{d t} \phi_{j}=g
$$

with

$$
g(t)=\sum_{|\vec{k}|^{2}=\Lambda_{j}} e^{-\frac{i \beta k_{1}}{\Lambda_{j}} t} \hat{f}_{\vec{k}} e^{i \vec{k} \cdot \vec{x}}
$$

where

$$
g \in L^{1}, \text { since }\|g(t)\|_{0}=\|f(t)\|_{0}
$$

Now the long time behavior of the shifted problem is easily derived. Let

$$
\phi_{\infty}=\phi_{j}\left(t_{0}\right)+\int_{t_{0}}^{\infty} g(s) d s
$$

we have the convergence of the shifted problem as

$$
\left\|\phi_{j}(t)-\phi_{\infty}\right\|_{0} \leq c t^{\frac{1}{2}} e^{-\nu \Lambda_{j} t / 2}
$$

To translate the result back to our original variable we assume that $\phi_{\infty}$ has the representation

$$
\phi_{\infty}=\sum_{|\vec{k}|^{2}=\Lambda_{j}} \hat{\phi}_{\infty, \vec{k}} e^{i \vec{k} \cdot \vec{x}}
$$

and we define

$$
\xi_{j}(t)=\sum_{|\vec{k}|^{2}=\Lambda_{j}} e^{\frac{i \beta k_{1}}{\Lambda_{j}} t} \hat{\phi}_{\infty, \vec{k}} e^{i \vec{k} \cdot \vec{x}}
$$

Notice that $\xi_{j}$ solves the equation

$$
\frac{d}{d t} \Delta \xi_{j}+\beta \frac{\partial \xi_{j}}{\partial x}=0
$$


and satisfies

$$
\left(I-P_{\Lambda_{j}}\right) \xi_{j}=0
$$

and

$$
\left\|\nabla \xi_{j}(t)\right\|_{0}=\left\|\nabla \psi_{\infty}\right\|_{0}
$$

Thus we have the following convergence result

$$
\left\|e^{\nu \Lambda_{j} t} \psi_{j}(t)-\xi_{j}(t)\right\|_{0}^{2} \leq c t^{\frac{1}{2}} e^{-\nu \Lambda_{j} t / 2}
$$

which further implies

$$
e^{\nu \Lambda_{j} t}\left\|\nabla \psi_{j}(t)\right\|_{0} \rightarrow \lim _{t \rightarrow \infty}\left\|\nabla \xi_{j}(t)\right\|_{0}=\left\|\nabla \phi_{\infty}\right\|_{0}
$$

or

$$
e^{\nu \Lambda_{j} t}\|\nabla \psi\|_{0}=\left\|\nabla \phi_{\infty}\right\|_{0}+\mathbf{o}(1)
$$

To complete the proof of our main theorem it remains to prove

$$
\left\|\frac{\psi_{j}(t)}{\|\nabla \psi(t)\|_{0}}-\frac{\xi_{j}(t)}{\left\|\nabla \psi_{\infty}\right\|_{0}}\right\|_{0} \rightarrow 0,
$$

since all norms on $E_{k}$ are equivalent.

Our estimates of the decay of motion off the $j_{t h}$ shell implies

$$
\|\nabla \psi\|_{0}=\left\|\nabla \psi_{j}\right\|_{0}+\text { l.o.t. }
$$

where l.o.t. represents lower order terms.

Thus

$$
\frac{\nabla \psi_{j}(t)}{\|\nabla \psi(t)\|_{0}}=\frac{e^{\nu \Lambda_{j} t} \nabla \psi_{j}(t)}{e^{\nu \Lambda_{j} t}\|\nabla \psi\|_{0}} \rightarrow \frac{\nabla \xi_{j}(t)}{\left\|\nabla \phi_{\infty}\right\|_{0}}
$$

This completes the proof of the main theorem with

$$
\vec{v}_{j}=\frac{\nabla^{\perp} \xi_{j}}{\left\|\nabla \phi_{\infty}\right\|_{0}} .
$$

To summaries, we have established the following

1. Selective decays states are eigenfunctions of the Laplacian.

2. Selective decays states are invariant under the barotropic quasi-geostrophic dynamics.

3. All solutions converge to the superposition of a Rossby wave and a shear flow, both of them consists of selective decay states.

4. Hyper-viscosity enhances the selective decay process.

5 . The $\beta$ plane generates Rossby wave.

Thus the only thing left is to study the stability of the ground shell and the instability of higher shells. The stability of the ground shell is a well-known result (see for instance the lecture notes of Majda and Embid, 1995). We restate the theorem here for the sake of completeness. 
THEOREM 2. Let $E_{k}$ be the $k^{\text {th }}$ shell, i.e., the eigenspace associated with the eigenvalue $\Lambda_{k}$. Then $E_{k}$ is unstable for $k>1$

Proof. Let $\vec{w}_{k}$ be a selective decay state on the $k^{\text {th }}$ shell, i.e.,

$$
\vec{w}_{k} \in E_{k} .
$$

We now consider a small perturbation from lower eigenspaces(normalized):

$$
\vec{w}_{j} \in E_{j}, \text { with } j<k
$$

Then for $\varepsilon$ small we have

$$
\begin{aligned}
\Lambda\left(\vec{w}_{k}+\varepsilon \vec{w}_{j}\right) & =\frac{\Lambda_{k}\left\|\vec{w}_{k}\right\|_{0}^{2}+\varepsilon^{2} \Lambda_{j}}{\left\|\vec{w}_{k}\right\|_{0}^{2}+\varepsilon^{2}} \\
& =\Lambda_{k}+\frac{\varepsilon^{2}}{\left\|\vec{w}_{k}\right\|_{0}^{2}+\varepsilon^{2}}\left(\Lambda_{j}-\Lambda_{k}\right) \\
& <\Lambda_{k} .
\end{aligned}
$$

Thus the limit of the Dirichlet quotient of the solution starting from $\vec{w}_{k}+\varepsilon \vec{w}_{j}$ must be something less than $\Lambda_{k}$ since the Dirichlet quotient is non-increasing. This proves the instability of $E_{k}, k>1$. This completes the proof of the theorem.

\section{REFERENCES}

[1] Brezis, H., AND Gallouet, T., Nonlinear Schrödinger evolution equations, Nonlinear Anal., TMA 4(1980), pp. 677-681.

[2] Constantin, P., And Foias, C., Navier-Stokes Equations, Chicago University Press, Chicago, 1988.

[3] Constantin, P., Foias, C. and Temam, R., 1988, On the dimension of the attractors in two-dimensional turbulence, Physica D, 30(1988), pp. 284-296.

[4] Eydeland, A., Spruck, J., ANd Turkington, B., Multiconstrained variational problems of nonlinear eigenvalue type: new formulations and algorithms, Math. Comput., 55(1990), pp. 509-535.

[5] FoiAs, C., AND SAUt, Asymptotic behaviour, as $t \rightarrow \infty$ of solutions of Navier-Stokes equations and non-linear spectral manifolds, Indiana Univ. Math. J., 33 (1984), pp. 459-477.

[6] Foias, C., AND Temam, R., Gevrey class regularity for the solutions of the Navier-Stokes equations, J. Funct. Anal., 87 (1989), pp. 359-369.

[7] Folland, G., Introduction to Partial Differential Equations, Princeton University press, Princeton, NJ, 1976.

[8] Holen, M., Comparison of statistical theories for large scale two-dimensional flows, Ph.D. thesis, Dept. of Mathematics, Princeton University, 1995, supervised by A. Majda.

[9] Majda, A., AND Bertozzi, A., Vorticity and the Mathematical theory of Incompressible Flow, To appear, 1999.

[10] MajdA, A., AND EmBid, P., Lecture Notes on PDE from Atmosphere/Ocean Sciences, CIMS, New York Univ., 1995.

[11] Majda, A., AND Holen, M., Dissipation, topography, and statistical theories for large scale coherent structure, CPAM, Vol. L (1998), pp. 1183-1234.

[12] Matthaeus,W.H., Stribling, W.T., Martinez, D., Oughton, S., And Montgomery, D., Decaying two-dimensional Navier-Stokes turbulence at very long times, Physica D, 51 (1991), pp. 531-538.

[13] Montgomery, D., Shan, X., Matthaeus, W. H., Navier-Stokes relaxation to sinh-Poisson states at finite Reynolds numbers, Phys. Fluids A, 5 (1993), No. 9, pp. 2207-2216.

[14] Montgomery, D., And Joyce, G., Statistical mechanics of negative temperature states, Phys. Fluids, 17 (1974), pp. 1139-1145.

[15] Pedlosky, J., Geophysical Fluid Dynamics, Springer-Verlag, Berlin and New York, 1979.

[16] Reed, M., And Simon, B., Methods of Modern Mathematical Physics, Academic Press, New York, 1980. 
[17] SHIm, S-Y., Ph. D Thesis (Supervised by A. Majda), CIMS, NYU, 1999.

[18] Temam, R., Navier-Stokes Equations and Nonlinear Functional Analysis, CBMS-NSF Regional Conference Series in Applied Mathematics, SIAM, Philadelphia (1983).

[19] Ting, A. C., Chen, H. H., AND LeE, Y. C., Exact solutions of a nonlinear boundary value problem: the vortices of the two-dimensional Sinh-Poisson equation, Physica D, 26 (1987), pp. 37-66.

[20] XIN, Z. P., Decay of the Dirichlet Quotient in the Presence of Hyper-Viscosity, Unpublished (1997). 